\title{
Feasibility and Safety of Left Atrial Appendage Closure Using the LAmbre Device in Patients with Nonvalvular Atrial Fibrillation with or Without Prior Catheter Ablation A Single Centre Experience
}

\author{
Xiang-Fei Feng, ${ }^{1}$ MD, Peng-Pai Zhang, ${ }^{1} \mathrm{PhD}$, Jian Sun, ${ }^{1} \mathrm{PhD}$, \\ Qun-Shan Wang, ${ }^{1} \mathrm{PhD}$ and Yi-Gang Li, ${ }^{1} \mathrm{MD}$
}

\begin{abstract}
Summary
Left atrial appendage (LAA) closure (LAAC) has emerged as an alternative therapeutic approach to medical therapy for stroke prevention in patients with nonvalvular atrial fibrillation (NVAF). However, complex LAA anatomy may preclude its use. LAmbre is a new, self-expanding LAA occluder, and is highly adaptable to different LAA morphologies. We explored the feasibility, safety, and efficacy of LAAC using LAmbre device in NVAF patients with or without prior catheter ablation (CA). LAAC using LAmbre device was applied in NVAF patients with (group C) or without (group N) prior CA. Transesophageal echocardiography (TEE) was performed at 3, and 12 months post-LAAC. Among 17 LAAC patients (group C, 6 \& group N, 11), 4 cases were implanted with special type devices, 5 were implanted with large devices. Besides one case of cardiac tamponade (N group), there were two minor peri-procedural complications only. Successful sealing of the LAA was documented in all the patients (100\%) by TEE both post LAAC and at 3 months. At 3 months, no residual flow was achieved in 11 patients $(64.7 \%)$; six patients $(35.3 \%)$ had residual flow $<5 \mathrm{~mm}$. There was no device dislocation or leakage during the mean of 30 months follow up. At 545 days after LAAC, one patient in group C experienced sudden death. Baseline, peri-procedural, and follow-up characteristics were similar between two groups $(P>0.05)$. LAAC with LAmbre device, subsequent to prior $\mathrm{CA}$ for $\mathrm{AF}$, can be performed successfully and safely. The design and distinguishing features of this device could be of help in patients with complex anatomy of LAA.
\end{abstract}

Key words: Special type device, Complication, Residual flow, Occlusion, Stroke

(Int Heart J 2019; 60: 63-70)

A trial fibrillation (AF) is the most common arrhythmia. Stroke is the most devastating complication of AF, accounting for $15 \%$ to $20 \%$ of all strokes. ${ }^{1)}$ Oral anticoagulation (OAC) is indicated to prevent thromboembolic events. The left atrial appendage is the primary source for thrombus formation and cardiac embolism in patients with non-valvular $\mathrm{AF}^{2)}$ Left atrial appendage device closure (LAAC) can be a meaningful alternative to OAC for patients who could not tolerate $\mathrm{OAC}$, and is increasingly applied clinically for stroke prevention in AF patients.,

Several studies have indicated that catheter ablation (CA) provides efficacious rhythm control for patients with symptomatic and drug refractory $\mathrm{AF}^{5,6)}$ and might be beneficial for reducing the risk of stroke..$^{7-9)}$ However, other studies have shown that patients may continue to have asymptomatic episodes of AF after apparently successful $\mathrm{CA}^{10)}$ and the risk of stroke remains high in these patients. ${ }^{11)}$ Thus, OAC should not be discontinued post-CA in higher risk patients. ${ }^{12)}$

Recently, as a two-pronged strategy, the combination of LAAC with CA in a single session, has been tested as a comprehensive way to improve the symptoms while at the same time reducing the incidence of stroke without the need to take OAC in selected high-risk AF patients. Clinical studies have confirmed that hybrid procedure (CA plus LAAC) could be performed successfully and safely in a single procedure, with a lower than expected stroke rate. ${ }^{13-15)}$ In addition, previous studies have showed that CA following prior LAAC was feasible and efficacious, and was not linked with intra-procedural or periprocedural device interference, dislocation, or leakage. ${ }^{16,17)}$ However, clinical observation on the safety and efficacy of LAAC in nonvalvular AF (NVAF) patients with prior CA is scanty now.

Currently, Watchman and Amplatzer Cardiac Plug are the two mostly implanted LAAC devices worldwide and numerous studies have demonstrated the satisfactory clini-

From the ${ }^{1}$ Department of Cardiology, Xinhua Hospital, School of Medicine, Shanghai Jiao Tong University, Shanghai, China.

This study was supported by Lifetech Scientific Corp., Ltd, Shenzhen, China.

Address for correspondence: Yi-Gang Li, MD, Department of Cardiology, Xinhua Hospital, School of Medicine, Shanghai Jiao Tong University, 1665\#,

KongJiang Road, Shanghai 200092, China. E-mail: liyigang@xinhuamed.com.cn

Received for publication January 30, 2018. Revised and accepted April 23, 2018.

Released in advance online on J-STAGE November 20, 2018

doi: 10.1536/ihj.18-070

All rights reserved by the International Heart Journal Association. 
cal results of these two devices. LAmbre ${ }^{\mathrm{TM}}$ is a new, selfexpanding LAA occluder constructed from a nitinol mesh and frame with polyester membranes. It consists of an umbrella and a cover connected by a short central waist. ${ }^{18)}$ Until now, clinical data on LAmbre ${ }^{\mathrm{TM}}$ device use is rare. ${ }^{19,20)}$ In the present study, we reported the feasibility and safety of LAAC with LAmbre in the presence or absence of prior CA procedures.

\section{Methods}

Patient selection: The previous trial ${ }^{21)}$ (clinicaltrials.gov identifier NCT02029014) was an open-label, nonrandomized, prospective, multicenter study, which has demonstrated the safety, feasibility, and efficacy of deploying the LAmbre LAA occlusion device for stroke prevention in AF. This study population is composed of all patients performed in Xinhua Hospital Affiliated to Shanghai Jiao Tong University, School of Medicine for the trial. Patients with symptomatic NVAF referring for LAAC with or without prior CA from August to December 2014 were included in this study. This study was approved by the institutional review board for human research and complied with the Declaration of Helsinki. Since LAAC was not reimbursed in China, the procedures were financed by a Research Funding grant, up to a maximum of 18 procedures per year.

Pulmonary venous atrium isolation (PVAI) ablation was performed in paroxysmal AF patients and stepwise ablation in non-paroxysmal AF patients. ${ }^{22}$ The risks of stroke and bleeding were determined according to the $\mathrm{CHADS}_{2}$ and the HAS-BLED scores. The indications for LAAC were NVAF, over 18 years of age, with an increased risk of stroke $\left(\mathrm{CHADS}_{2}\right.$ score $\left.\geq 1\right)$, a contraindication for and /or failure of OAC (e.g., embolic event in spite of adequate OAC). ${ }^{23)}$ As described in detail previously, ${ }^{21}$ the exclusion criteria were as follows: symptomatic patients with carotid artery disease, left ventricular ejection fraction $<30 \%$, acute myocardial infarction or unstable angina, acute infective endocarditic, stroke or transient ischemic attack within 30 days, cardiac tumors or other malignancy with estimated life expectancy $<2$ years, presence of thrombus in the heart, pregnancy, presence of a prosthetic valve, LAA orifice diameter $\leq 12$ $\mathrm{mm}$, a known allergy to nitinol. All the patients were evaluated by an electrophysiologist in the outpatient clinic for eligibility, and all the participants signed a written consent form. Transesophageal echocardiography (TEE) was performed to assess LAA anatomy, and exclude LAA thrombus and significant structural cardiac abnormalities such as congenital absence ${ }^{24)}$ in all the patients within 1 week before the LAAC procedure. All the data were collected prospectively by means of a web-based database.

LAAC procedure: LAAC was performed with the LAmbre $^{\mathrm{TM}}$ device (Lifetech Scientific Corp., Shenzhen, China). The details of the device and procedure have been described elsewhere. ${ }^{18)}$ Briefly, all the procedures were guided by TEE, and after a single transseptal puncture by use of the modified Brockenbrough technique and an $8 \mathrm{Fr}$ $\mathrm{SL}_{1}$ transseptal sheath (St. Jude Medical), systemic anticoagulation was made with intravenous heparin $(100 \mathrm{u} / \mathrm{kg}$, bolus) to maintain an activated clotting time of $300 \mathrm{sec}-$ onds. Then, the $\mathrm{SL}_{1}$ transseptal sheath was exchanged for the appropriate delivery sheath. A 5 Fr pigtail catheter was advanced into the LAA via the delivery sheath, and LAA angiograms were performed. The diameters of the orifice and length of the LAA were measured from the respective LAA angiograms (right anterior oblique $30^{\circ}$ and caudal $20^{\circ}$ ).

Then, the delivery sheath containing the device was placed on the proximal part of the LAA. The umbrella of the device was partially deployed by slowly pushing out from the delivery sheath. Thereafter, the whole system was gently pushed "en-bloc" forward to the desired landing zone to allow for better flowering of the umbrella and grasping of the LAA walls by the retention hooks. Then, the sheath was withdrawn to expose the cover, allowing it to expand in the left atrium and covering the LAA ostium by gently pushing the delivery cable forward. Once the device was placed in the LAA, left atrial angiogram and TEE were performed to check the device positioning, LAA sealing, and impingement on surrounding cardiac structures. A gentle tug test by applying tension to the delivery cable was performed to ensure the device stability. The device would be released from the delivery cable once acute procedural success was achieved, or the device would be intentionally retrieved and redeployed. After the release of the device, TEE was performed to check further.

After the procedure, all the patients were continuously monitored and received intravenous heparin (10000 $\mathrm{u}$, continuous infusion) for 24 hours, and then, both aspirin (100 mg daily) and clopidogrel (75 mg daily) were either resumed or started as standard of care. The patients were discharged on the following day after X-ray and TTE to exclude device dislocation, embolization, and pericardial effusion.

Follow-up: The patients were followed up by their treating electrophysiologist in the outpatient clinic at 1, 3, 6, and 12 months after the procedure. To evaluate the device position, residual flow, and thrombus formation, TEE was performed at 3 months and 12 months.

All the patients were prescribed aspirin and clopidogrel for 3 months after the procedure. After 3 months, the patients with successful sealing were then only switched to aspirin indefinitely unless contraindicated. OAC was prescribed if the criteria for successful sealing were not met, and in the patients with thrombus formation on left atrium or device. Long-term follow-up was advised for the patients as indicated.

Discretion: Acute procedural success is defined as proper and stable implant in the LAA without peri-device leakage (unsuccessful sealing) or impingement on surrounding cardiac structure. ${ }^{25}$ Sealing was considered successful when there was either no flow (complete) or the presence of a minimal residual flow of $<5 \mathrm{~mm}^{26}$ ) on the contrary, unsuccessful sealing was considered when a remaining jet was $\geq 5 \mathrm{~mm}$ or in an unsatisfactory position such as impingement on surrounding cardiac structures. Owing to the unsatisfactory position, or remaining jet, the device was retrieved and repositioned, or even exchanged until successfully deploy. The redeploy count and exchanged 


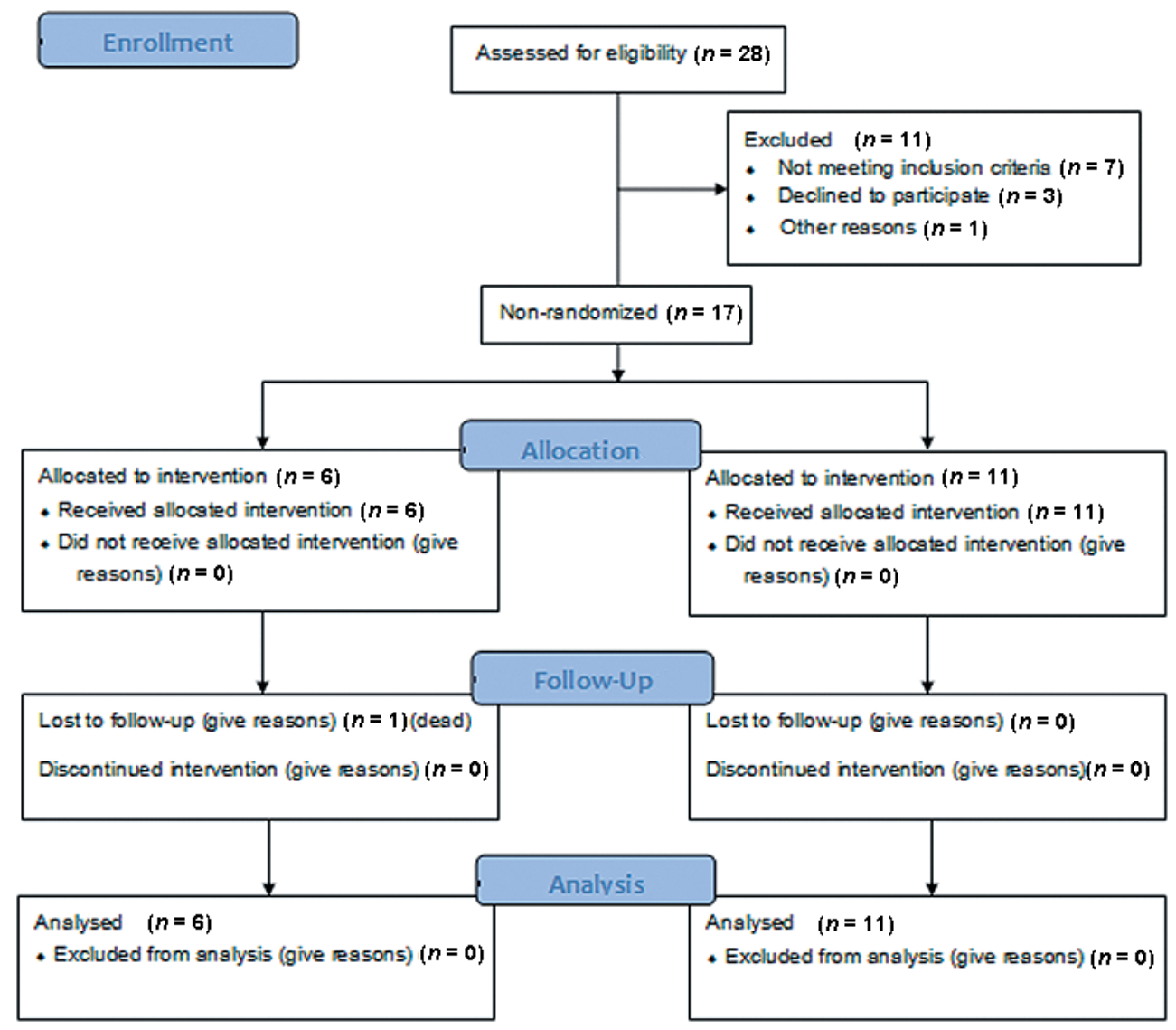

Figure 1. Flow diagram.

device were documented.

The definitions and reporting requirements for adverse event (AE) and serious $\mathrm{AE}(\mathrm{SAE})$ are based on a previous report and are defined in the study protocol. ${ }^{27}$ Major adverse events were defined as death, stroke, systemic embolism, and major bleedings requiring invasive treatment or blood transfusion. ${ }^{21)}$ Ischemic stroke was defined as the sudden onset of a focal neurological deficit in the distribution of a single brain artery with symptoms and/or signs persisting $\geq 24$ hours, or when $\leq 24$ hours if accompanied by the evidence of tissue loss without hemorrhage based on computed tomography or magnetic resonance brain imaging. ${ }^{28)}$ Freedom from left atrial arrhythmias was described after a blanking period of 3 months. ${ }^{13)}$

Two parameters are required for the decision-making of device selection: the cover diameter and the umbrella diameter. The former would be $4-8 \mathrm{~mm}$ larger than the measured LAA orifice, based on the clinical judgment of the implanting physician, ${ }^{18)}$ and the latter is usually 4 to 6 $\mathrm{mm}$ larger in diameter than landing zone. The LAA landing zone diameter is used for the selection of the device size. ${ }^{25)}$

The general type device is used if the cover is 4 to 6 $\mathrm{mm}$ larger in diameter than the umbrella. ${ }^{18)}$ Otherwise, if it is $>6 \mathrm{~mm}$, i.e., the device has a large cover with a small umbrella, the special type device (a large cover with a small umbrella) is used. A large device is defined as the diameter of cover $\geq 35 \mathrm{~mm}$.

Statistics: Descriptive statistics were used to report the patient characteristics. Continuous variables with normal distribution were reported as mean \pm SD. Median (25th to 75 th percentiles) was used with abnormal distribution. Percentages were used to report categorical variables. All the statistical analyses were performed in SPSS software (version 22.0, SPSS Inc., Chicago, Illinois).

\section{Results}

Baseline characteristics: Eleven out of the 28 screened patients were excluded from the study according to the exclusion criteria. Consequently, 18 patients were registered in the LAAC cohort. One of these patients was excluded from the analysis because of LAAC with Watchman device. Finally, 17 consecutive patients with a history of symptomatic NVAF were analyzed (Figure 1). The mean age was $71.4 \pm 7.8$ years, and seven patients (41.2\%) were male. Hypertension was present in all the patients. Persistent AF, ischemic stroke, diabetes mellitus, congestive heart failure, percutaneous transluminal coronary intervention, and frequent ventricular premature contraction (VPC) (> 1,000 per 24 hours $^{29)}$ ) were found in 14 patients $(82.4 \%), 8$ patients $(47.1 \%), 2$ patients $(11.8 \%), 3$ patients $(17.6 \%)$, and 2 patients $(11.8 \%), 1$ patients $(5.9 \%)$ respectively. 
Table I. Baseline Characteristics in 17 Patients with Procedure of LAmbre ${ }^{\mathrm{TM}}$ LAAC $(n=17)$

\begin{tabular}{lcccc}
\hline & Total $(n=17)$ & Group C $(n=6)$ & Group N $(n=11)$ & $P$ value \\
\hline Age (years) & $71.4 \pm 7.8$ & $68.5 \pm 6.3$ & $72.9 \pm 8.3$ & 0.278 \\
Male, $n(\%)$ & $7(41.2 \%)$ & $1(16.7 \%)$ & $6(54.5 \%)$ & 0.317 \\
Ischemic stroke, $n(\%)$ & $8(47.1 \%)$ & $3(50.0 \%)$ & $5(45.5 \%)$ & 1.000 \\
Diabetes mellitus, $n(\%)$ & $2(11.8 \%)$ & $1(16.7 \%)$ & $1(9.10 \%)$ & 1.000 \\
Hypertension, $n(\%)$ & $17(100 \%)$ & $6(100 \%)$ & $11(100 \%)$ & - \\
Frequent VPC, $n(\%)$ & $1(5.9 \%)$ & $1(16.7 \%)$ & $0(0.00 \%)$ & 0.751 \\
HF, $n(\%)$ & $3(17.6 \%)$ & $1(16.7 \%)$ & $2(18.2 \%)$ & 1.000 \\
PCI, $n(\%)$ & $2(11.8 \%)$ & $1(16.7 \%)$ & $1(9.10 \%)$ & 1.000 \\
CHADS 2 score & $2.5 \pm 1.1$ & $2.3 \pm 1.2$ & $2.6 \pm 1.0$ & 0.592 \\
HAS-BLED score & $2.7 \pm 0.8$ & $3.0 \pm 0.9$ & $2.5 \pm 0.7$ & 0.259 \\
Pro-BNP (pg/mL) & $760.9 \pm 1005.7$ & $780.3 \pm 793.4$ & $750.4 \pm 1141.6$ & 0.696 \\
LVEF $(\%)$ & $63.6 \pm 4.4$ & $62.7 \pm 5.1$ & $64.1 \pm 4.1$ & 0.537 \\
Persistent AF, $n(\%)$ & $16(94.1 \%)$ & $5(83.3 \%)$ & $11(100 \%)$ & 0.353 \\
\hline
\end{tabular}

Pro-BNP indicates brain natriuretic peptide; LVEF, left ventricular ejection fraction; HF, congestive heart failure; PCI, percutaneous transluminal coronary intervention; VPC, ventricular premature contraction; and $\mathrm{AF}$, atrial fibrillation.

Among the 17 patients, six patients (35.3\%) with prior CA were defined as group C, 11 patients $(64.7 \%)$ without prior CA were defined as group N. Dense spontaneous echo contrast in the left atrium was found in 8 patients $(47.1 \%$ ) by TEE ( 2 in group C, 6 in group $N$ ) and LAA angiogram, the mean diameter of the LAA "neck" was $20.9 \pm 3.6 \mathrm{~mm}$ at the landing zone and $25.9 \pm 5.3$ $\mathrm{mm}$ at the ostium. A large ostium $(>30 \mathrm{~mm})$ was seen in four patients $(23.5 \%)$, and multi-lobed LAA was seen in 13 patients $(76.5 \%)$.

Among 6 patients in group C, 5 cases were nonparoxysmal AF and had previously stepwise CA, another one was paroxysmal AF and had had PVAI previously, while in group $\mathrm{N}$, all the 11 cases were non-paroxysmal AF. The demographic and baseline characteristics were similar between the two groups (all $P>0.05$ ) (Table I).

Procedural characteristics: In all the 17 patients, 13 cases were implanted with general type devices and 4 with special type devices (a large cover with a small umbrella) (Figure 2). Among the 17 devices, 5 were large (cover diameter $\geq 35 \mathrm{~mm}$ ), and all the LAmbre ${ }^{\mathrm{TM}}$ devices were implanted successfully (100\%). LAAC was achieved with a mean procedure time of $67.2 \pm 11.9$ minutes, fluoroscopy time of $18.6 \pm 14.4$ minutes, redeploy count of $1.76 \pm 1.03$ times. Device implantation was managed with the first device selected in $94 \%$ (16) of the successful cases, and in $5.9 \%$ ( 1 case), a second device was needed. The device exchange count was $0.06 \pm 0.24$ times. By TEE after the device was released, two patients had residual flow $<5 \mathrm{~mm}$, other cases were documented as no residual flow, which indicated successful sealing of all the LAA (100\%).

During the time of the procedure, the device was retrieved and repositioned for four times in one patient owing to the unsatisfactory position, which resulted in cardiac tamponade managed successfully by emergency pericardiocentesis. Then, the patient was switched to a smaller device $($ diameter $=32 / 36 \mathrm{~mm}$ ) and LAAC was successful at the end.

Besides the cardiac tamponade in 1 patient, there were 2 other minor peri-procedure complications related to procedure: one patient developed a small tongue hematoma, and one patient developed a small groin hematoma. All the patients were discharged the next day after X-ray and TTE confirmation of the satisfactory device positioned in the LAA. Table II summarizes the procedural parameters.

From Table II, we can see that the total procedure time, fluoroscopy time, redeploy count, device exchange count, and peri-procedure complications were not significantly different between the two groups (all $P>0.05$ ).

Follow up results at 90 days and one-year post procedure: Successful sealing of the LAA was documented in all the patients $(100 \%)$ at 3 months post LAAC by TEE. Complete sealing was seen in 11 cases $(64.7 \%)$, small residual flow $<5 \mathrm{~mm}$ was seen in six other patients $(35.3 \%)$, and there was no thrombus on the device. Repeated TEE at 12 months showed complete sealing in additional four patients, and minimal residual flow of $<5$ $\mathrm{mm}$ was seen in the remaining two patients $(18.2 \%)$. Neither residual flow $\geq 5 \mathrm{~mm}$ nor asymptomatic device embolization/thrombus was observed and the success rate of LAAC remained as $100 \%$ during the 12 months follow-up (Table III).

Safety: In the present study, seven patients experienced 15 SAEs within 12 months post LAAC, of which one was deemed by the investigators to be related to the device or the implant procedure $(5.9 \%)$. No clinical neurological or embolic events were detected based on patient symptom reporting during follow-up to date since device implantation. During the mean follow-up period of 30 months, none of the patients had thrombus formation on the surface of the device or left atrium; however, dense spontaneous echo contrast in the left atrium was documented in four patients $(23.5 \%, 3$ at 12 months, 1 at 22 months) (Table III). Another patient used clopidogrel instead of aspirin due to having had gastrorrhagia previously. Three patients experienced minor procedural or device unrelated bleeding event (Table II). In group $\mathrm{C}$, a patient with minimal residual flow $2.5 \mathrm{~mm}$ at peri-procedure period died suddenly at 545 days after LAAC (Table III).

From Table III, we can see that the residual flow, 


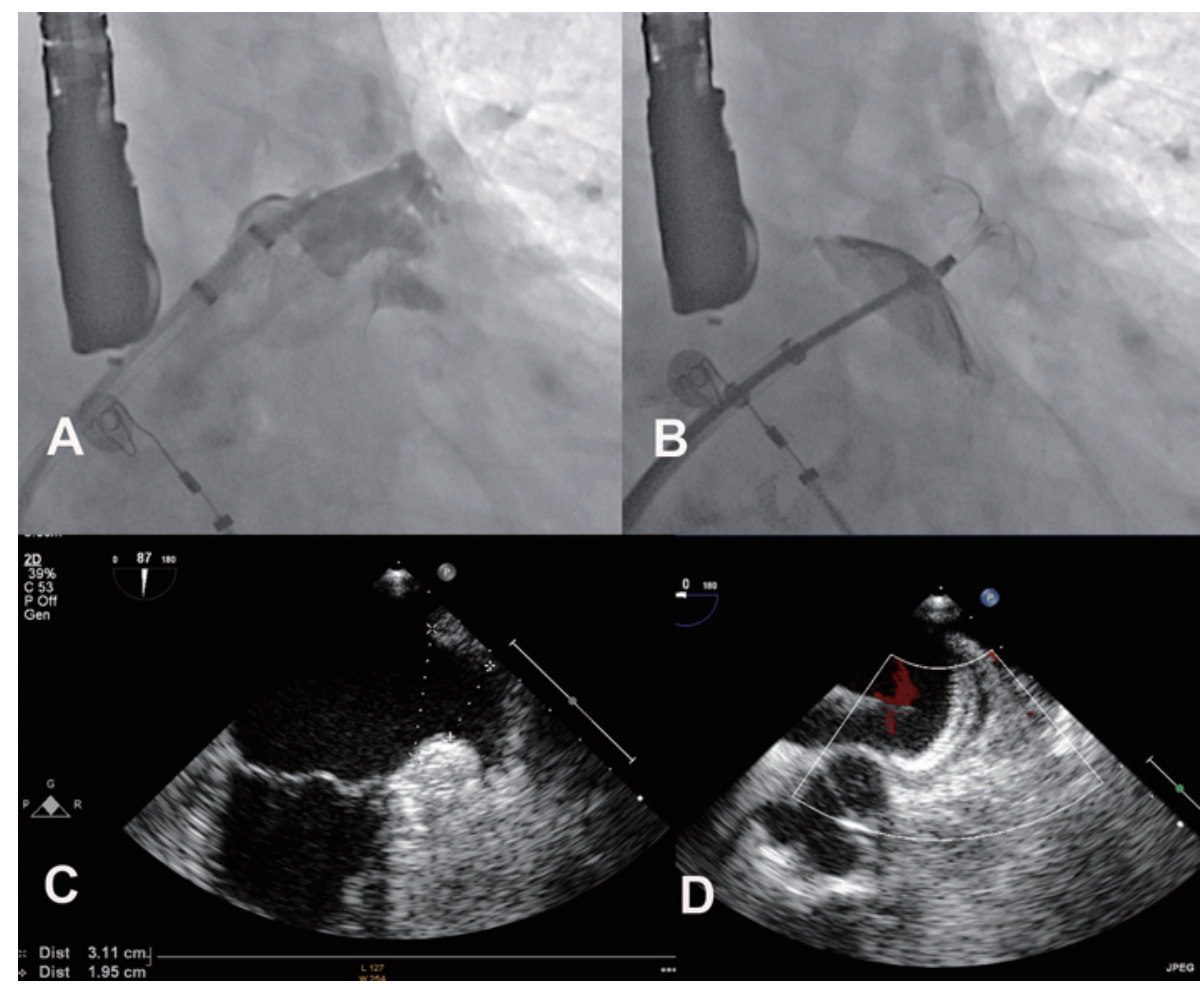

Figure 2. Fluoroscopy and TEE of LAA closure with a special type LAmbre device (22/34 mm). A: LAA angiography before closure. B: LAA angiography after closure with a special type device well-positioned in the LAA. C: TEE examination before LAA closure. D: TEE examination after LAA closure. Angiographic and TEE examinations suggest that a special type LAmbre device completely occluded the LAA, and there is no significant peridevice leak. LAA indicates left atrial appendage; and TEE, transesophageal echocardiography.

Table II. Procedural Characteristics in Patients with LAAC Procedure $(\bar{\chi} \pm$ SD $)(n=17)$

\begin{tabular}{lcccc}
\hline & Total $(n=17)$ & Group C $(n=6)$ & Group N $(n=11)$ & $P$ value \\
\hline LAA ostium width $(\mathrm{mm})$ & $25.7 \pm 5.5$ & $24.4 \pm 5.1$ & $26.7 \pm 5.4$ & 0.412 \\
LAA length (mm) & $29.1 \pm 6.7$ & $28.1 \pm 8.6$ & $27.4 \pm 4.9$ & 0.855 \\
Landing zone (mm) & $21.5 \pm 4.0$ & $22.1 \pm 5.4$ & $20.3 \pm 2.2$ & 0.330 \\
LA diameter (mm) & $45.6 \pm 5.3$ & $44.4 \pm 6.1$ & $44.7 \pm 5.1$ & 0.547 \\
Multi-lobed LAA, $n(\%)$ & $13(76.5 \%)$ & $4(66.7 \%)$ & $9(81.8 \%)$ & 0.916 \\
Dense spontaneous echo contrast, $n(\%)$ & $8(47.1 \%)$ & $2(33.3 \%)$ & $6(54.5 \%)$ & 0.742 \\
Umbrella Size (mm) & $26.9 \pm 4.9$ & $28.0 \pm 6.1$ & $24.4 \pm 3.2$ & 0.122 \\
Cover Size (mm) & $32.9 \pm 4.6$ & $33.0 \pm 5.5$ & $32.9 \pm 4.3$ & 0.773 \\
Procedure Time (minutes) & $67.2 \pm 11.9$ & $69.5 \pm 11.2$ & $66.0 \pm 12.6$ & 0.578 \\
Fluoroscopic Time (minutes) & $18.8 \pm 12.2$ & $19.3 \pm 11.5$ & $18.1 \pm 13.8$ & 0.432 \\
Redeploy Count (times) & $1.76 \pm 1.03$ & $2.0 \pm 1.1$ & $1.6 \pm 1.0$ & 0.506 \\
Case with Device Exchange, $n(\%)$ & $1(5.90 \%)$ & $1(16.7 \%)$ & $0(0.00 \%)$ & 0.353 \\
No Residual Flow, $n(\%)$ & $15(88.2 \%)$ & $4(66.7 \%)$ & $11(100 \%)$ & 0.110 \\
Residual Flow < $<$ mm, $n(\%)$ & $2(11.8 \%)$ & $2(33.3 \%)$ & $0(0.00 \%)$ & 0.110 \\
Residual Flow $\geq 5$ mm, $n(\%)$ & $0(0.00 \%)$ & $0(0.00 \%)$ & $0(0.00 \%)$ & - \\
Cardiac Tamponade, $n(\%)$ & $1(5.90 \%)$ & $0(0.00 \%)$ & $1(9.10 \%)$ & 0.647 \\
Minor bleeding event, $n(\%)$ & $2(11.8 \%)$ & $1(16.7 \%)$ & $1(9.1 \%)$ & 1.000 \\
\hline
\end{tabular}

LA indicates left atrial; and LAA, left atrial appendage

pericardial effusion, dense spontaneous echo contrast, and MAE were not significantly different between the two groups through 30 months of follow-up (all $P>0.05$ ).

\section{Discussion}

The results of this study demonstrate the feasibility and safety of LAmbre ${ }^{\mathrm{TM}}$ implant procedures, subsequent to prior CA, with satisfactory LAA occlusion maintained 
Table III. Follow-Up Characteristics During a Follow-Up Period of 30 Months in Patients with LAAC Procedure $(\bar{\chi} \pm$ SD) $(n$ $=17$ )

\begin{tabular}{|c|c|c|c|c|}
\hline & Total $(n=17)$ & Group C $(n=6)$ & Group $\mathrm{N}(n=11)$ & $P$ value \\
\hline Follow-Up Period (days) & $872 \pm 90$ & $830 \pm 142$ & $894 \pm 38$ & 0.324 \\
\hline Residual flow $<5 \mathrm{~mm}$ through 3 months, $n(\%)$ & $6(35.3 \%)$ & $3(50.0 \%)$ & $3(27.3 \%)$ & 0.685 \\
\hline Pericardial effusion through 3 months, $n(\%)$ & $5(29.4 \%)$ & $2(33.3 \%)$ & $3(27.3 \%)$ & 1.000 \\
\hline Pericardial effusion through 12 months, $n(\%)$ & $0(0.00 \%)$ & $0(0.00 \%)$ & $0(0.00 \%)$ & - \\
\hline Thrombus on device through 3 months, $n(\%)$ & $0(0.00 \%)$ & $0(0.00 \%)$ & $0(0.00 \%)$ & - \\
\hline Device malposition through 3 months, $n(\%)$ & $0(0.00 \%)$ & $0(0.00 \%)$ & $0(0.00 \%)$ & - \\
\hline Device malposition through 12 months, $n(\%)$ & $0(0.00 \%)$ & $0(0.00 \%)$ & $0(0.00 \%)$ & - \\
\hline Death during $30 \mathrm{Ms}$ follow-up, $n(\%)$ & $1(5.90 \%)$ & $1(16.7 \%)$ & $0(0.00 \%)$ & 0.353 \\
\hline Stroke or TIA through 30 months, $n(\%)$ & $0(0.00 \%)$ & $0(0.00 \%)$ & $0(0.00 \%)$ & - \\
\hline Device embolization through 30 months, $n(\%)$ & $0(0.00 \%)$ & $0(0.00 \%)$ & $0(0.00 \%)$ & - \\
\hline
\end{tabular}

TIA indicates transient ischemic attack.

at follow-up.

Ablation-induced tissue injury at the ostium of the LAA following left atrial CA was frequently observed on TEE during the procedures, ${ }^{14)}$ and maybe affected LAAC. However, LAAC combined with CA for AF can be performed successfully and safely in a single procedure, with a lower than expected stroke rate. ${ }^{15)}$

Maybe the injured LAA ostium tissue caused by prior CA will change in nature or structure or will be linked with intra-procedural, peri-procedural device interference, dislocation, or leakage. However, the data about that is rare. Our data showed that both in group $\mathrm{C}$ and group $\mathrm{N}$, the ostium width and length of LAA, landing zone, LA diameter were not significantly different (all $P>$ 0.05 ). In other words, prior CA did not cause a change in the structure of LAA. According to our data, the total procedure time, fluoroscopy time, redeploy count, device exchange count, and peri-procedure complications were not significantly different between two groups. In other words, prior CA did not increase the difficulty and risk of LAAC.

Recently, percutaneous LAAC devices were introduced for stroke prevention in patients with NVAF, and their safety and feasibility have been demonstrated in large clinical trials. ${ }^{30)}$ In addition, recent data have shown that complete LAAC by device resulted in a reduction of AF burden, specifically in patients with proven LAA ectopy. ${ }^{31)}$ Therefore, LAAC could be an effective alternative to $\mathrm{OAC}$ for stroke prevention in patients with non-valvular $\mathrm{AF}^{3)}$

LAmbre $^{\mathrm{TM}}$ is a novel, self-expanding LAAC device. Its main advantages include a small delivery system and the ability to be fully retrievable and repositionable during implantation. The avoidance of deep seating of the delivery catheter into the LAA during deployment can potentially reduce the risk of LAA perforation. ${ }^{18)}$

Data, especially clinical data, about LAmbre ${ }^{\text {TM }}$ device is rare. To the best of our knowledge, this is the first report of LAAC with the LAmbre ${ }^{\mathrm{TM}}$ device in patients with prior CA.

Our data also showed that a $100 \%$ success rate of implanting LAmbre ${ }^{\mathrm{TM}}$ device and a $100 \%$ adequate LAA sealing rate were achieved in both group $\mathrm{C}$ and group $\mathrm{N}$. In this case series, no clinical thromboembolic events were detected in a cohort with a mean $\mathrm{CHADS}_{2}$ score of $2.5 \pm 1.1$ over a follow-up time of up to 30 months.

Although LAAC is becoming a new-emerging procedure worldwide for stroke prevention in AF patients, there are still some anatomic features of LAA, which significantly limits its use. Since the most popular devices do not allow the closure of very large LAAs, the size of the LAA is one of the current limitations. The LAmbre ${ }^{\mathrm{TM}}$ device is highly adaptable to different LAA morphologies and, thus, it can be very useful in difficult anatomies, such as chicken wing anatomies with a very short implantation zone or conical LAA with a large size difference between the ostium and the medial part of the LAA. The combination of distal hooks, the U-shaped ends, and the central waist design of LAmbre ${ }^{\mathrm{TM}}$ may be helpful to achieve complete sealing and to prevent embolization in complex cases. Our data showed the successful experience of special type devices used in four patients, large device used in five patients, indicating the superiority of LAmbre $^{\text {TM }}$ design.

Study limitations: The present study has several limitations, including nonrandomized single-center study and the small number of patients enrolled in this study. The follow-up assessments for embolic events were detected based on patients' symptom and, thus, the occurrence rate might be underestimated. TEE assessment was relatively invasive and low-throughput, ${ }^{32)}$ and was performed at 3 and 12 months after LAAC and, therefore, transient thrombus formation in the interval phase after LAAC cannot be excluded. However, the relatively long follow-up time makes the data solid to interpret key events. In the current study, stepwise CA, more extensive ablation in the left atrium, might increase the possibility of iatrogenic damage to the ostium of LAA. However, the data about that is rare. Thus, more clinical trials are needed to confirm the iatrogenic damage further. 


\section{Conclusions}

LAA closure with LAmbre ${ }^{\mathrm{TM}}$ device, subsequent to prior CA, is safe and feasible for stroke prevention in symptomatic NVAF patients. The design and distinguishing features of this device could be of help in patients with complex anatomy of LAA.

\section{Disclosures}

Conflicts of interest: The authors report no relationships that could be construed as a conflict of interest.

\section{References}

1. De Backer O, Arnous S, Ihlemann N, et al. Percutaneous left atrial appendage occlusion for stroke prevention in atrial fibrillation: an update. Open Heart 2014; 1: e000020.

2. Blackshear JL, Odell JA. Appendage obliteration to reduce stroke in cardiac surgical patients with atrial fibrillation. Ann Thorac Surg 1996; 61: 755-9.

3. Holmes DR Jr, Kar S, Price MJ, et al. Prospective randomized evaluation of the Watchman Left Atrial Appendage Closure device in patients with atrial fibrillation versus long-term warfarin therapy: the PREVAIL trial. J Am Coll Cardiol 2014; 64: 1-12.

4. Lewalter T, Kanagaratnam P, Schmidt B, et al. Ischaemic stroke prevention in patients with atrial fibrillation and high bleeding risk: opportunities and challenges for percutaneous left atrial appendage occlusion. Europace 2014; 16: 626-30.

5. Tzou WS, Marchlinski FE, Zado ES, et al. Long-term outcome after successful catheter ablation of atrial fibrillation. Circ Arrhythm Electrophysiol 2010; 3: 237-42.

6. Calkins H, Kuck KH, Cappato R, et al. 2012 HRS/EHRA/ ECAS expert consensus statement on catheter and surgical ablation of atrial fibrillation: recommendations for patient selection, procedural techniques, patient management and follow-up, definitions, endpoints, and research trial design: a report of the Heart Rhythm Society (HRS) Task Force on Catheter and Surgical Ablation of Atrial Fibrillation. Developed in partnership with the European Heart Rhythm Association (EHRA), a registered branch of the European Society of Cardiology (ESC) and the European Cardiac Arrhythmia Society (ECAS); and in collaboration with the American College of Cardiology (ACC), American Heart Association (AHA), the Asia Pacific Heart Rhythm Society (APHRS), and the Society of Thoracic Surgeons (STS). Endorsed by the governing bodies of the American College of Cardiology Foundation, the American Heart Association, the European Cardiac Arrhythmia Society, the European Heart Rhythm Association, the Society of Thoracic Surgeons, the Asia Pacific Heart Rhythm Society, and the Heart Rhythm Society. Heart Rhythm 2012; 9: 632-96.e21.

7. Gaita F, Sardi D, Battaglia A, et al. Incidence of cerebral thromboembolic events during long-term follow-up in patients treated with transcatheter ablation for atrial fibrillation. Europace 2014; 16: 980-6.

8. Reynolds MR, Gunnarsson CL, Hunter TD, et al. Health outcomes with catheter ablation or antiarrhythmic drug therapy in atrial fibrillation: results of a propensity-matched analysis. Circ Cardiovasc Qual Outcomes 2012; 5: 171-81.

9. Kochhäuser S, Alipour P, Haig-Carter T, et al. Risk of stroke and recurrence after AF ablation in patients with an initial event-free period of 12 months. J Cardiovasc Electrophysiol 2017; 28: 273-9.

10. Verma A, Champagne J, Sapp J, et al. Discerning the incidence of symptomatic and asymptomatic episodes of atrial fibrillation before and after catheter ablation (DISCERN AF): a prospec- tive, multicenter study. JAMA Intern Med 2013; 173: 149-56.

11. Wilber DJ, Pappone C, Neuzil P, et al. Comparison of antiarrhythmic drug therapy and radiofrequency catheter ablation in patients with paroxysmal atrial fibrillation: a randomized controlled trial. JAMA 2010; 303: 333-40.

12. Calkins H, Kuck KH, Cappato R, et al. 2012 HRS/EHRA/ ECAS Expert Consensus Statement on Catheter and Surgical ablation of atrial fibrillation: recommendations for patient selection, procedural techniques, patient management and follow-up, definitions, endpoints, and research trial design. Europace 2012; 14: 528-606.

13. Swaans MJ, Post MC, Rensing BJ, Boersma LV. Ablation for atrial fibrillation in combination with left atrial appendage closure: first results of a feasibility study. J Am Heart Assoc 2012; 1: e 002212

14. Phillips KP, Walker DT, Humphries JA. Combined catheter ablation for atrial fibrillation and Watchman(r) left atrial appendage occlusion procedures: five-year experience. J Arrhythm 2016; 32: $119-26$.

15. Calvo N, Salterain N, Arguedas H, et al. Combined catheter ablation and left atrial appendage closure as a hybrid procedure for the treatment of atrial fibrillation. Europace 2015; 17: 153340.

16. Walker DT, Phillips KP. Left atrial catheter ablation subsequent to Watchman(r) left atrial appendage device implantation: a single centre experience. Europace 2015; 17: 1402-6.

17. Heeger CH, Rillig A, Lin T, et al. Feasibility and clinical efficacy of left atrial ablation for the treatment of atrial tachyarrhythmias in patients with left atrial appendage closure devices. Heart Rhythm 2015; 12: 1524-31.

18. Lam YY. A new left atrial appendage occluder (Lifetech LAmbre Device) for stroke prevention in atrial fibrillation. Cardiovasc Revasc Med 2013; 14: 134-6.

19. Cruz-Gonzalez I, Moreno-Samos JC, Rodriguez-Collado J, Mo Y, Lam YY, Sanchez PL. Percutaneous closure of left atrial appendage with complex anatomy using a LAmbre device. JACC Cardiovasc Interv 2017; 10: e37-9.

20. Zhou Q, Song H, Zhang L, et al. Roles of real-time threedimensional transesophageal echocardiography in peri-operation of transcatheter left atrial appendage closure. Medicine (Baltimore) 2017; 96: e5637.

21. Huang $\mathrm{H}$, Liu Y, Xu Y, et al. Percutaneous left atrial appendage closure with the LAmbre device for stroke prevention in atrial fibrillation: A prospective, multicenter clinical study. JACC Cardiovasc Interv 2017; 10: 2188-94

22. Xiang FF, Yi Y, Zhang R, Sun J, Zhang PP, Lu SB, Yi Gang Li. Left atrial pressure, proBNP and hsCRP during periablation period in atrial fibrillation patients. Int J Clin Exp Med 2016; 9: $2777-85$

23. Koneru JN, Badhwar N, Ellenbogen KA, Lee RJ. LAA ligation using the LARIAT suture delivery device: tips and tricks for a successful procedure. Heart Rhythm 2014; 11: 911-21.

24. Enomoto Y, Hashimoto G, Sahara N, et al. Congenital absence of left atrial appendage diagnosed by multimodality imaging. Int Heart J 2018; 59: 439-42.

25. Lam YY, Yan BP, Doshi SK, et al. Preclinical evaluation of a new left atrial appendage occluder (Lifetech LAmbre device) in a canine model. Int J Cardiol 2013; 168: 3996-4001.

26. Holmes DR, Reddy VY, Turi ZG, et al. Percutaneous closure of the left atrial appendage versus warfarin therapy for prevention of stroke in patients with atrial fibrillation: a randomised noninferiority trial. Lancet 2009; 374: 534-42.

27. Boersma LV, Schmidt B, Betts TR, et al. Implant success and safety of left atrial appendage closure with the WATCHMAN device: peri-procedural outcomes from the EWOLUTION registry. Eur Heart J 2016; 37: 2465-74.

28. Gangireddy SR, Halperin JL, Fuster V, Reddy VY. Percutaneous left atrial appendage closure for stroke prevention in patients with atrial fibrillation: an assessment of net clinical benefit. Eur Heart J 2012; 33: 2700-8. 
29. Wilber DJ. Ventricular ectopic beats: not so benign. Heart 2009; 95: 1209-10.

30. Belgaid DR, Khan Z, Zaidi M, Hobbs A. Prospective randomized evaluation of the watchman left atrial appendage closure device in patients with atrial fibrillation versus long-term warfarin therapy: the PREVAIL trial. Int J Cardiol 2016; 219: 1779.

31. Afzal MR, Kanmanthareddy A, Earnest M, et al. Impact of left atrial appendage exclusion using an epicardial ligation system (LARIAT) on atrial fibrillation burden in patients with cardiac implantable electronic devices. Heart Rhythm 2015; 12: 52-9.

32. Watanabe A, Suzuki S, Kano H, et al. Left atrial remodeling assessed by transthoracic echocardiography predicts left atrial appendage flow velocity in patients with paroxysmal atrial fibrillation. Int Heart J 2016; 57: 177-82. 